
\title{
R Research Square \\ The Expression Profiles and Clinical Significance of PKM2 and Notch1 in Colorectal Cancer
}

\section{Jia Wang}

Lanzhou University Second Hospital

Meijuan Sun

Baoji second People's Hospital

\section{Wenlong Du}

Lanzhou University Second Hospital

\section{Yuanxian Guo}

Lanzhou University Second Hospital

\section{Jiebin Pan}

Lanzhou University Second Hospital

\section{Bangguo Kou}

Lanzhou University Second Hospital

\section{Yongjie Jiang}

Lanzhou University Second Hospital

\section{Pan Bian}

Lanzhou University Second Hospital

\section{Bingtai Li}

Lanzhou University Second Hospital

Lanning Yin ( $\square$ yinlanning@163.com )

Lanzhou University Second Hospital

\section{Research}

Keywords: colorectal cancer, pyruvate kinase M2, Notch1, compound 3k, tangeretin, synergistic effect

Posted Date: September 28th, 2021

DOl: https://doi.org/10.21203/rs.3.rs-907818/v1

License: (9) This work is licensed under a Creative Commons Attribution 4.0 International License. Read Full License 


\section{The expression profiles and clinical significance of PKM2 and Notch1 in colorectal cancer}

Jia Wang ${ }^{1,2^{*}}$, Meijuan $\mathrm{Sun}^{3 *}$, Wenlong $\mathrm{Du}^{2 *}$, Yuanxian $\mathrm{Guo}^{2}$, Jiebin $\mathrm{Pan}^{2}$, Bangguo $\mathrm{Kou}^{2}$ Yongjie Jiang $^{2}$, Pan Bian ${ }^{2}$, Bingtai $\mathrm{Li}^{2}$, Lanning Yin ${ }^{2,4}$

1 Department of General Surgery, Baoji city People's Hospital, Baoji, China; 2 Department of General Surgery, Lanzhou University Second Hospital, Lanzhou, China; 3 Department of Pharmacy, Baoji Second People's Hospital, Baoji, China; 4 Department of General Surgery, Xigu District of Lanzhou City People's Hospital, Lanzhou, China.

* These authors contributed equally to this work.

Correspondence to: Lanning Yin. Department of General Surgery, Lanzhou University Second Hospital, No. 82 Cuiyingmen, Cheng-Guan District, Lanzhou, China; Department of General Surgery, Xigu District of Lanzhou City People's Hospital, No. 377, Fuli East Road, Xigu District, Lanzhou, China. Email: yinlanning@163.com.

\section{Abstract \\ Background}

Studies have shown that pyruvate kinase M2 (PKM2) and Notch1 are highly expressed in colorectal cancer (CRC) tissues and have a certain relationship with disease occurrence and development. The expression levels of PKM2 and Notch1 are also related to the effect of chemotherapy and radiotherapy, which seriously influence the prognosis of CRC patients. Thus, both PKM2 and Notch1 have been identified as key targets of CRC treatment and research. However, correlations between PKM2 and Notch1 have not yet been established.

\section{Methods}

Immunohistochemical analysis was conducted to detect the expression of PKM2 and Notch1 in CRC tissues. The mRNA and protein expression levels of PKM2 and Notch1 in CRC cell lines were detected by quantitative real-time fluorescence polymerase chain reaction (qRT-PCR) and western blot analysis, respectively. Compound $3 \mathrm{~K}$ and tangeretin (TGN) were used to inhibit the expression of PKM2 and Notch1, respectively. The proliferation and migration of cancer cells in each 
group were detected with the CCK-8 and wound healing assays.

\section{Results}

The Immunohistochemical analysis showed that PKM2 and Notch1 were highly expressed in CRC and related to tumor stage and lymph node metastasis. The qRTPCR and western blot results showed that PKM2 and Notch1 were notably upregulated in CRC cells both at the mRNA and protein levels. PKM2 and Notch1 form a positive feedback loop to promote the occurrence and development of CRC, and inhibition of PKM2 and Notch1 has a synergistic effect on the proliferative and invasive capabilities of CRC cells.

\section{Conclusion}

The combination of the PKM2 inhibitor compound 3K and the Notch1 inhibitor TGN presents a novel and effective strategy for treatment of CRC.

Keywords: colorectal cancer, pyruvate kinase M2, Notch1, compound 3k, tangeretin, synergistic effect

\section{Introduction}

Colorectal cancer (CRC) is the third most commonly diagnosed malignancy and the fourth leading cause of cancer-related death worldwide [1]. Without treatment, patients with inoperable or metastatic CRC have a median life expectancy of about 8 months [2]. Since the early stage of CRC is often asymptomatic, most patients are diagnosed in the mid to late stages of disease and, thus, miss the opportunity for surgery. Even if surgery is performed early, some patients will develop postoperative recurrence and metastasis. Therefore, chemotherapy is the primary treatment option for such patients. However, CRC cells are highly resistant to various chemotherapeutic drugs. Therefore, it is very important to develop novel drugs for the treatment of CRC.

Pyruvate kinase (PK), which is a key enzyme in glycolysis, catalyzes phosphoenolpyruvate into pyruvate and releases energy. Mammalian cells produce four isoforms of PK: L, R, M1, and M2 [3]. PKM2 is mainly expressed in embryonic cells, adult stem cells, and actively proliferating tissue cells. As the embryo develops, PKM2 is gradually replaced by several other isoenzymes. However, during 
tumorigenesis, PKM2 expression is increased and gradually replaces other isozyme types [4]. High expression of PKM2 in CRC tissues is predictive of a poor outcome [5]. Targeting of PKM2 with compound $3 \mathrm{~K}$ inhibits the development of CRC by suppressing glycolysis [6].

The Notch signaling pathway is a highly conserved intercellular signal transduction system that consists of the Notch receptor, Notch ligand, and transcription factor CSL. The Notch receptors Notch1-4 are transmembrane glycoproteins. Notch1 expression is closely correlated with poor overall survival of CRC patients and, thus, is a potential prognostic biomarker [7].

The aim of the current study was to investigate the relationship between the key lactate metabolism enzyme PKM2 and the Notch signaling pathway, and to determine whether simultaneous inhibition of PKM2 and Notch1 with compound 3K and tangeretin (TGN), respectively, exerts synergistic effects against CRC cells. The results suggest that this approach presents a novel strategy for treatment of CRC.

\section{Materials and Methods}

\section{Study approval and patient consent}

The study protocol was approved by the Ethics Committee of Lanzhou University Second Hospital (Lanzhou, China) and conducted in accordance with the ethical principles for medical research involving human subjects as described in the Declaration of Helsinki. Written informed consent was obtained from each patient prior to surgery.

\section{Tissue samples}

CRC tissues $(n=66)$ were collected from patients (age range, 34-80 years; mean age, 60.3 years) who underwent surgery in Lanzhou University Second Hospital between March 2018 and February 2019. A diagnosis of CRC was confirmed by an experienced pathologist. None of the patients received chemotherapy or radiation therapy before resection.

\section{Immunohistochemical analysis}

The CRC tissues were fixed, dehydrated, embedded in paraffin, and then cut into $4 \mu \mathrm{m}$-thick slices, which were stained with hematoxylin. Prior to analysis, the tissue specimens were deparaffinized using a graded series of xylene and rehydrated with a 
graded series of ethanol. Antigen retrieval was performed using sodium citrate and blocking was performed using $\mathrm{H}_{2} \mathrm{O}_{2}$. The sections were then incubated with primary antibodies against PKM2 and Notch1 for $16 \mathrm{~h}$ at $4^{\circ} \mathrm{C}$ and then with horseradish peroxidase-conjugated secondary antibody (Beijing Zhongshan Jinqiao Biological Technology Co., Ltd., Beijing, China) for $2 \mathrm{~h}$ at room temperature. The presence of the secondary antibody was detected using a Cell and Tissue Staining Horseradish Peroxidase-Diaminobenzidine Kit (Beijing Zhongshan Jinqiao Biological Technology Co., Ltd.). An orthophoto microscope was used to obtain images of the stained tissue specimens.

All of the stained tissue specimens were graded by two qualified pathologists using a two-level scoring method. The proportion of positively stained cancer cells of each section was counted and the degree of staining was quantified. The grades of the stained tissue specimens are shown in Table 1. Finally, the results of the two scores were multiplied. A score $<3$ indicated negative protein expression, while a score of $\geq 3$ indicated positive expression.

Table 1. Immunohistochemical staining scoring system

\begin{tabular}{rrcr}
\hline $\begin{array}{c}\text { Percentage of positively } \\
\text { stained cells }\end{array}$ & Score & Degree of staining & Score \\
\hline$<5 \%$ & 0 & Unstained & 0 \\
$5 \% \sim 25 \%$ & 1 & Faint yellow & 1 \\
$26 \% \sim 50 \%$ & 2 & Yellowish brown & 2 \\
$51 \% \sim 75 \%$ & 3 & Brown & 3 \\
$>75 \%$ & 4 & & \\
\hline
\end{tabular}

\section{Cell culture and drugs}

Normal human colon epithelial NCM460 cells were purchased from Hunan Fenghui Biotechnology Co., Ltd. (Hunan, China). Human CRC RKO and HT29 cells were purchased from the Shanghai Institute of Cell Biology (Chinese Academy of Sciences, Shanghai, China). All cells were culured in Roswell Park Memorial Institute 1640 medium (Gibco, Carlsbad, CA, USA) supplemented with 10\% fetal bovine serum (Gibco) under a humidified atmosphere of $5 \% \mathrm{CO}_{2} / 95 \%$ air at $37^{\circ} \mathrm{C}$. The PKM2 inhibitor compound 3K was purchased from Shanghai Selleck Chemicals Co., Ltd. (Wuhan, China) and the Notch1 inhibitor TGN was purchased from 
MedChemExpress LLC (Monmouth Junction, NJ, USA). Antibodies against PKM2 were purchased from Affinity Biosciences (Cincinnati, OH, USA), those against Notch1 were obtained from Abcam (Cambridge, MA, USA), and those against glyceraldehyde 3-phosphate dehydrogenase (GAPDH) were procured from Proteintech (Wuhan, China).

\section{Cell Counting Kit-8 (CCK-8) assay}

The RKO and HT29 cells were plated in the wells 96-well plates at a density of 5000 cells/well and treated with dimethyl sulfoxide (DMSO) as a control, compound $3 \mathrm{~K}$ ( $9 \mu \mathrm{M}$ for RKO cells and $6 \mu \mathrm{M}$ for HT29 cells), TGN ( $25 \mu \mathrm{M})$, or the combination of compound $3 \mathrm{~K}$ and TGN. After $24 \mathrm{~h}, 10 \mu \mathrm{L}$ of CCK-8 reagent were added to each well and the optical density (OD) at $450 \mathrm{~nm}$ was measured using a microplate reader. The cell proliferation rate (\%) was calculated as (OD experimental group-OD CCK8)/(OD control group - OD CCK-8).

\section{Wound healing assay}

RKO and HT29 cells $\left(5 \times 10^{5}\right)$ were seeded in the wells of six-well plates. When the cells reached $>95 \%$ confluence, wounds were created using a $200-\mu 1$ and $10-\mu 1$ sterile pipette tip for the RKO and HT29 cells, respectively. After washing away the floating cells with phosphate-buffered saline and replacing the medium, DMSO (as a control), compound 3K ( $9 \mu \mathrm{M}$ for RKO cells and $6 \mu \mathrm{M}$ for HT29 cells), TGN (25 $\mu \mathrm{M}$ ), or a combination of compound $3 \mathrm{~K}$ and TGN was added to the wells. The wound healing results, indicating migration ability, were recorded for up to $24 \mathrm{~h}$ using an optical microscope.

\section{Quantitative real-time fluorescence polymerase chain reaction (qRT-PCR)}

Total RNA was extracted from NCM460, RKO, and HT29 cells using TRIzol reagent (Takara Bio, Inc., Shiga, Japan) and reverse transcribed into complementary DNA using a PrimeScript RT Reagent Kit (Takara Bio, Inc.). The amplifications were performed using SYBR Green Master Mix (Yeasen, Shanghai, China) with genespecific primers. $\beta$-actin was used as an internal control. The primers were as follows:

PKM2（F): 5'- CAGAGGCTGCCATCTACCAC -3'

PKM2（R): 5'- GACGAGCTGTCTGGGGATTC -3'

Notch1（F): 5'- TACAAGTGCGACTGTGACCC -3' 
Notch1（R): 5'- ATACACGTGCCCTGGTTCAG -3'

$\beta$-actin (F): 5'-TGGCACCCAGCACAATGAA-3'

$\beta$-actin ( R ): 5'-CTAAGTCATAGTCCGCCTAGAAGCA-3'。

\section{Western blot analysis}

Total proteins were extracted from NCM460, RKO, and HT29 cells by homogenization in radioimmunoprecipitation assay buffer containing phenylmethylsulfonyl fluoride. Equal amounts of proteins (30 $\mu \mathrm{g}$ per lane) were separated by electrophoresis using $10 \%$ sodium dodecyl sulfate gels and then transferred to polyvinylidene fluoride membranes, which were blocked with $5 \%$ nonfat milk in Tris-buffered saline with Tween 20 (TBST) at room temperature for $2 \mathrm{~h}$ and then incubated overnight at $4{ }^{\circ} \mathrm{C}$ with primary antibodies against PKM2, Notch1, and GAPDH. Afterward, the membranes were washed with TBST and incubated with horseradish peroxidase-conjugated goat anti-rabbit immunoglobulin $\mathrm{G}$ secondary antibodies. Protein bands were visualized using a ChemiDoc ${ }^{\mathrm{TM}}$ Gel Imaging System (Bio-Rad Laboratories, Hercules, CA, USA).

\section{Statistical analyses}

All statistical analyses were performed using IBM SPSS Statistics for Windows, version 26.0. (IBM Corporation, Armonk, NY, USA). Differences among groups were evaluated using one-way analysis of variance. A probability $(p)$ value of $<0.05$ was considered statistically significant.

\section{Results}

\section{PKM2 and Notch1 were upregulated in CRC tissues}

The result showed that PKM2 and Notch1 were highly expressed in $71.2 \%$ (47/66) and 68.2\% (45/66) of CRC tissues, respectively (Figure 1 and Table 2), and were clearly co-expressed (Table $3, \mathrm{R}=0.356, p<0.01$ ). Notably, there were significant differences between PKM2 and sex, age, lymph node metastasis, and tumor stage, and between Notch1 and lymph node metastasis and tumor stage. 
A

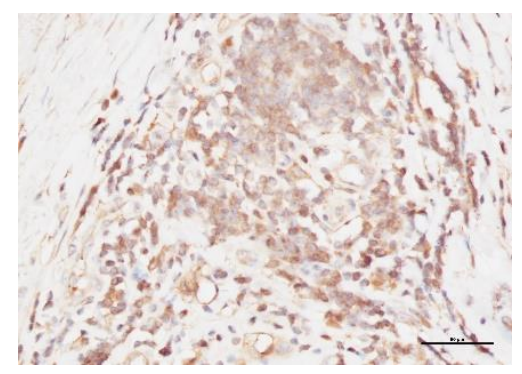

$\mathrm{C}$

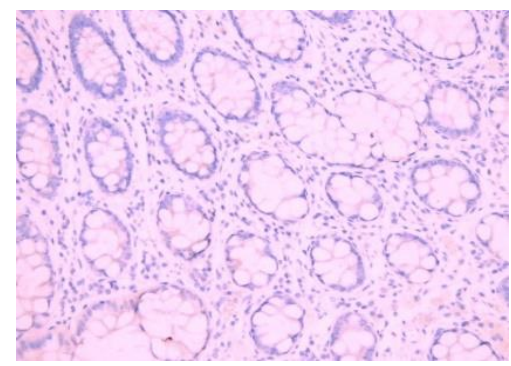

B

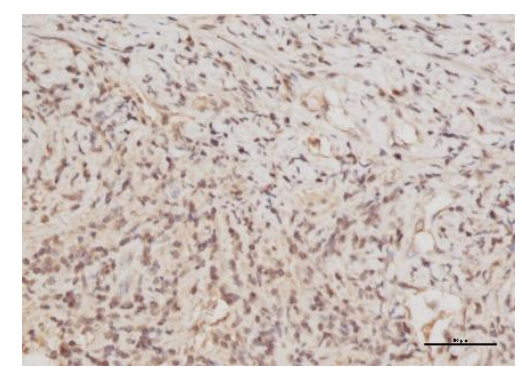

$\mathrm{D}$

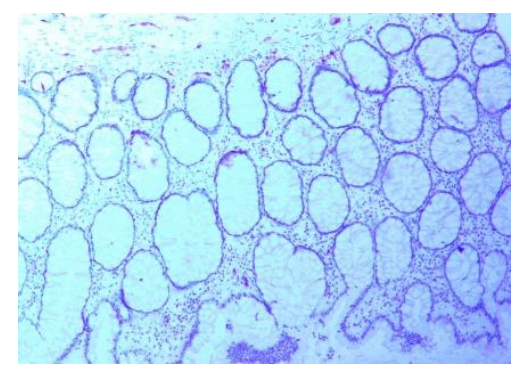

Figure 1. Immunohistochemistry. A and B show that positive expressions of PKM2 and Notch1 in CRC tissues, while $\mathrm{C}$ and $\mathrm{D}$ are negative expressions in normal tissues. (X200)

Table 2. Correlation between PKM2 and Notch1 expression in CRC

\begin{tabular}{lll}
\hline & PKM2 & Notch1 \\
\hline PKM2 & 1 & $0.356^{* *}$ \\
& - & 0.003 \\
Notch1 & 66 & 66 \\
& $0.356^{* *}$ & 1 \\
& 0.003 & - \\
& 66 & 66 \\
\hline
\end{tabular}

Table 3 Relationship between PKM2 and Notch1 expression and clinical features of CRC

\begin{tabular}{lllllllll}
\hline $\begin{array}{l}\text { Clinical } \\
\text { characteristics }\end{array}$ & & $\mathrm{n}$ & \multicolumn{2}{c}{ PKM2 } & P value & Notch1 & P value \\
\hline Sex & & & - & + & & - & + & \\
& Male & 40 & 7 & 33 & $0.012^{*}$ & 14 & 26 & 0.491 \\
& Female & 26 & 12 & 14 & & 7 & 19 & \\
Age (years) & & & & & & & & \\
& $<60$ & 31 & 4 & 7 & $0.016^{*}$ & 8 & 23 & 0.324 \\
& $\geq 60$ & 35 & 15 & 20 & & 13 & 22 & \\
Tumor stage & & & & & & & & \\
& I-II & 35 & 13 & 22 & $0.01^{*}$ & 15 & 20 & $0.041^{*}$
\end{tabular}




\begin{tabular}{lcclllllll}
\multicolumn{2}{r}{} & III-IV & 31 & 6 & 25 & & 6 & 25 & \\
\multicolumn{2}{l}{ Lymph node metastasis } & & & & & & & \\
& No & 36 & 14 & 22 & $0.047 *$ & 15 & 21 & $0.028^{*}$ \\
Yes & 30 & 5 & 25 & & 5 & 25 & \\
Tumor marker & & & & & & & & \\
CEA & $<5$ & 44 & 14 & 30 & 0.58 & 12 & 32 & 0.309 \\
& $\geq 5$ & 20 & 5 & 15 & & 8 & 12 & \\
CA199 & $<27$ & 45 & 13 & 32 & 0.83 & 13 & 32 & 0.531 \\
& $\geq 27$ & 19 & 6 & 3 & & 7 & 12 & \\
CA125 & $<40$ & 54 & 16 & 38 & 0.981 & 18 & 36 & 0.642 \\
& $\geq 40$ & 10 & 3 & 7 & & 2 & 8 & \\
\hline
\end{tabular}

\section{PKM2 and Notch1 were upregulated in human CRC cells}

In human CRC RKO and HT29 cells and normal human normal colorectal epithelial NCM460 cells, the mRNA, and protein levels of PKM2 and Notch1 were evaluated using qRT-PCR and western blot analysis, respectively. The results showed that PKM2 and Notch1 were notably upregulated in CRC cells both at the mRNA (Figure 2A and B) and protein (Figure 2C-F) levels.

A

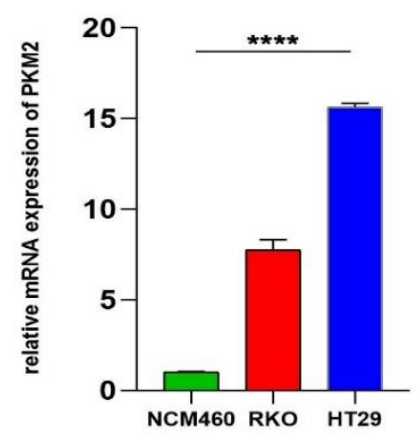

$\mathrm{C}$

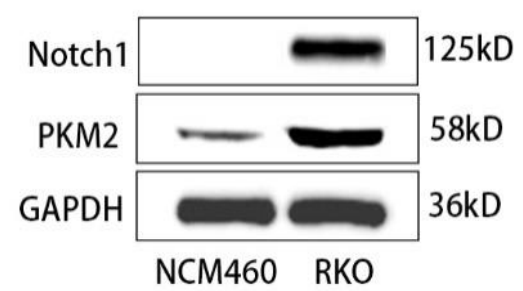

B

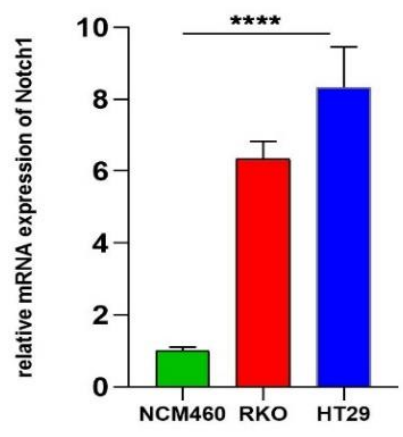

$\mathrm{D}$

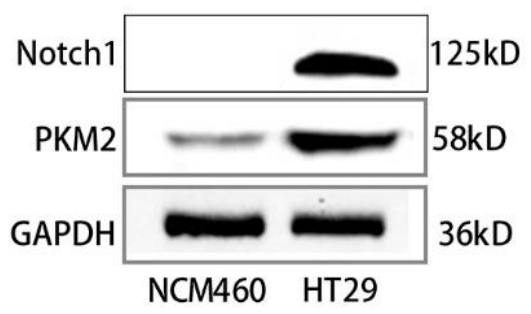


$\mathrm{E}$

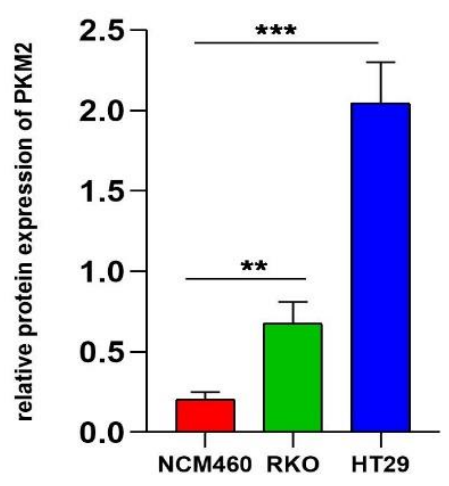

$\mathrm{F}$

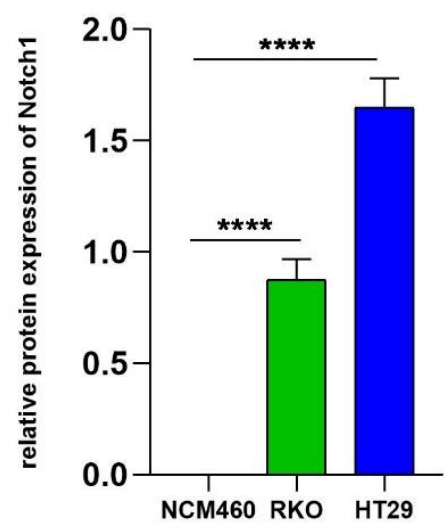

Figure 2. Expression of PKM2 and Notch1 in CRC cells. (A-B) qRT-PCR was performed to detect the mRNA expression levels of PKM2 and Notch1 in CRC cells. (C-F) Western blot analysis was performed to detect the protein expression levels of PKM2 and Notch1 in CRC cells. $* * p<0.01 ; * * * p<0.001 ; * * * * p<0.0001$.

\section{PKM2 and Notch1 affect the proliferation and mobility of CRC cells}

The CCK-8 results showed that suppression of PKM2 or Notch1 significantly reduced proliferation of RKO and HT29 cells (Figure 3A-C, $p<0.01$ ). The combination of compound $3 \mathrm{~K}$ and TGN significantly inhibited the proliferation of RKO and HT29 cells as compared to either individually and DMSO at $24 \mathrm{~h}$. The results of the wound healing assay showed that compound $3 \mathrm{~K}$ combined with TGN significantly inhibited the migration rate of RKO and HT29 cells as compared to either individually and DMSO (Figure 4A-C, $p<0.01$ ). Collectively, the results revealed that the PKM2 inhibitor compound $3 \mathrm{~K}$ and the Notch1 inhibitor TGN exerted synergistic effects on cell proliferation and mobility.

A

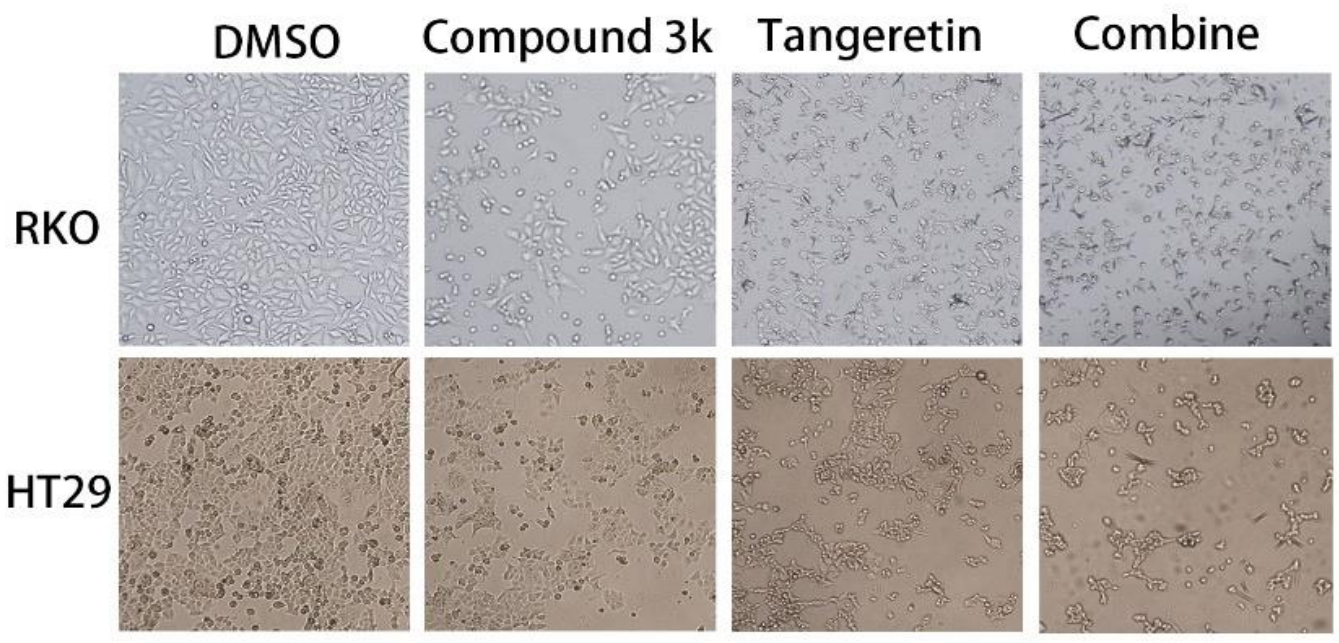


B

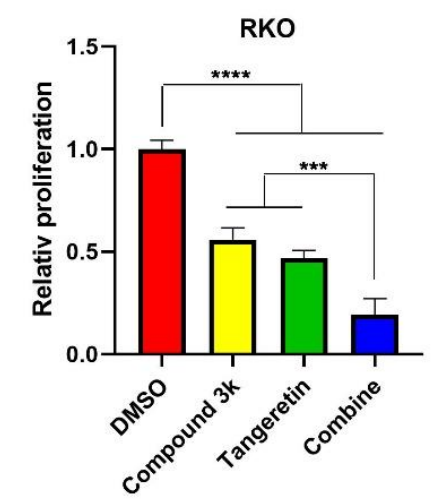

$\mathrm{C}$

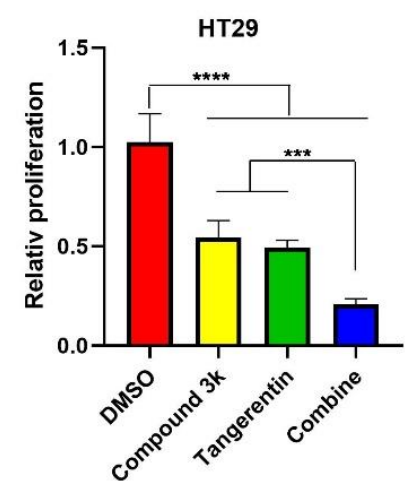

Figure 3. The PKM 2 inhibitor compound $3 \mathrm{~K}$ and the Notch1 inhibitor TGN exerted synergistic effects on the proliferation of RKO and HT29 cells. (A-C) The wound healing assay was performed to assess the proliferation of CRC cells after $24 \mathrm{~h}$ of treatment with DMSO (as a control), compound $3 \mathrm{~K}$, TGN, or the combination of compound $3 \mathrm{~K}$ and TGN. ${ }^{* * *} p<0.001$; $* * * * p<0.0001$.

A

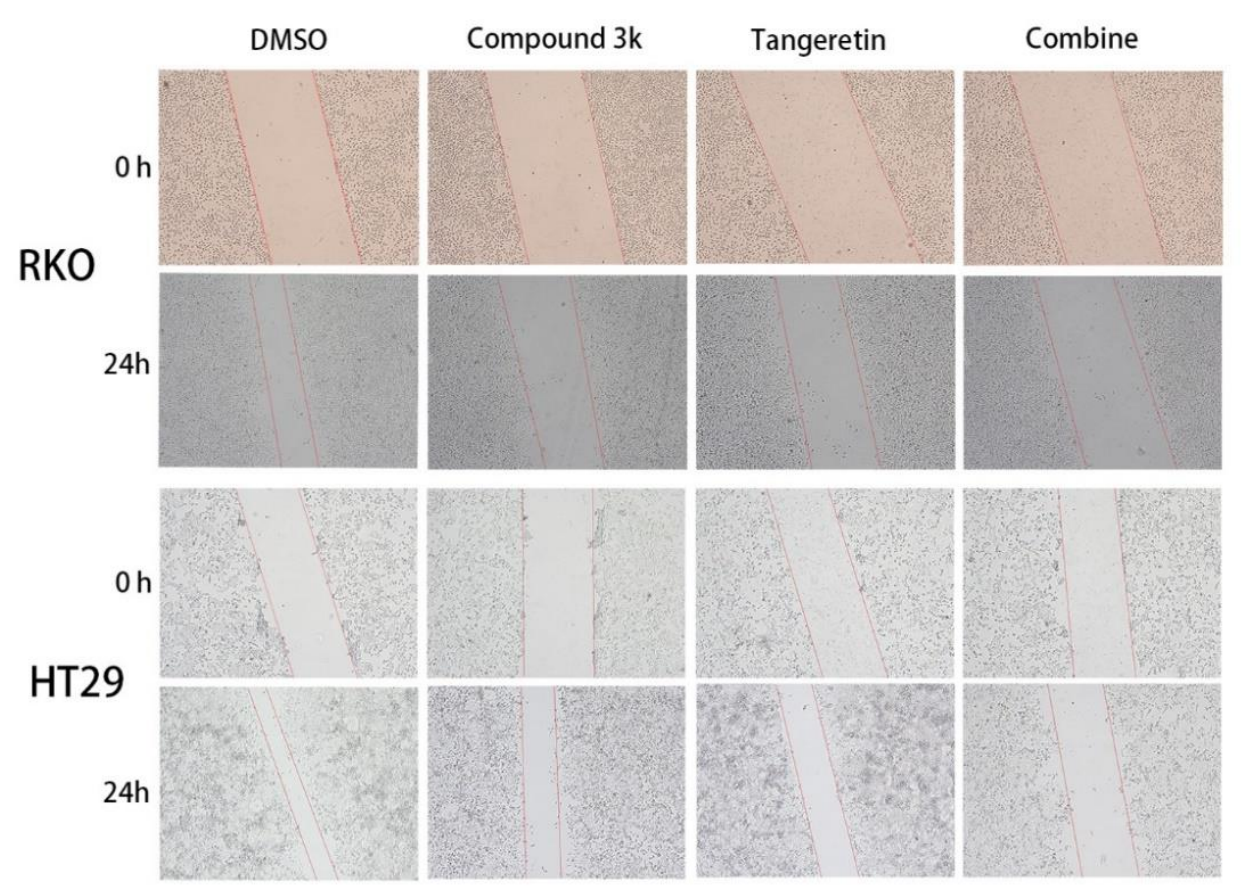


B

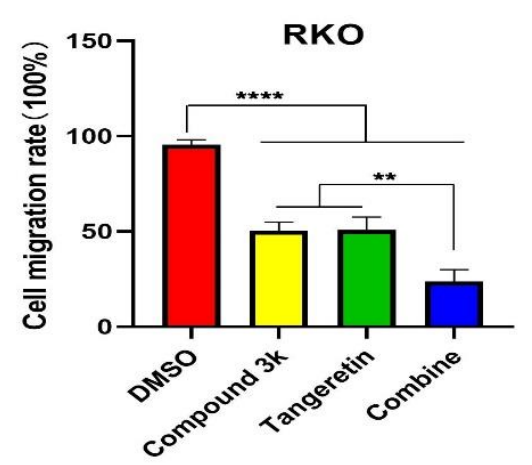

$\mathrm{C}$

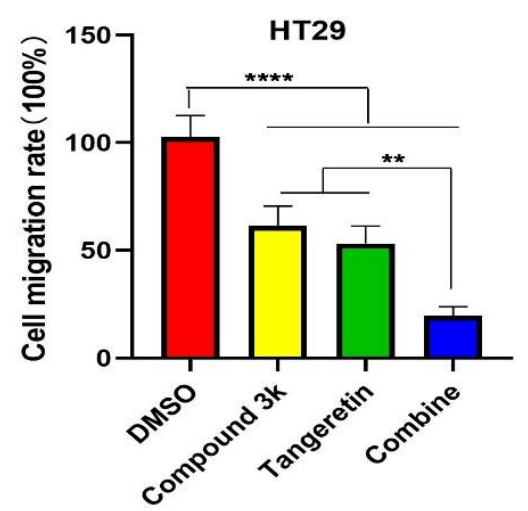

Figure 4. The PKM2 inhibitor compound $3 \mathrm{~K}$ and the Notch1 inhibitor TGN exerted synergistic effects on the mobility of RKO and HT29 cells. (A-C) The wound healing assay was performed to assess the migration of CRC cells after $24 \mathrm{~h}$ of treatment with DMSO (as a control), compound $3 \mathrm{~K}$, TGN, or the combination of compound $3 \mathrm{~K}$ and TGN. **p $<0.01$; **** $p<0.0001$.

\section{The relationship between PKM2 and Notch1 in CRC cells}

The results showed that suppression of PKM2 decreased the mRNA and protein levels of Notch1 in RKO and HT29 cells, while the combination of compound 3K and TGN exhibited stronger inhibitory effects as compared to either individually. Interestingly, suppression of Notch1 decreased the mRNA and protein levels of PKM2, and the combination of compound $3 \mathrm{~K}$ and TGN exhibited stronger inhibitory effects as compared to the single drugs. These results indicate that compound $3 \mathrm{~K}$ and TGN exert synergistic effects on expression of PKM2 and Notch1.

A

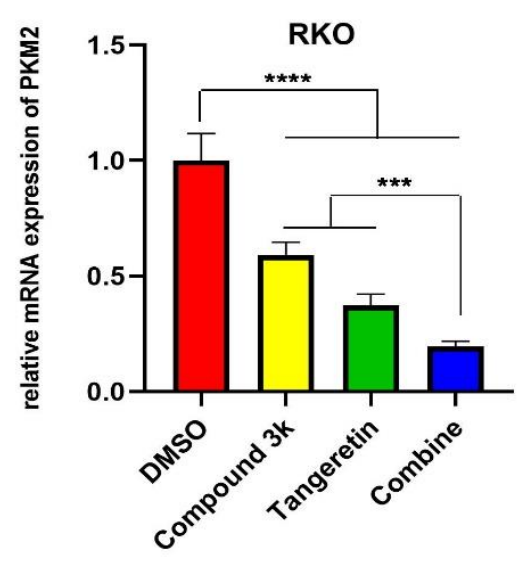

B

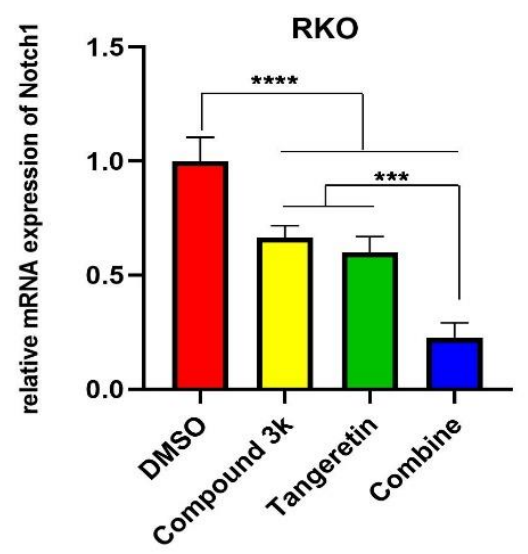


$\mathrm{C}$

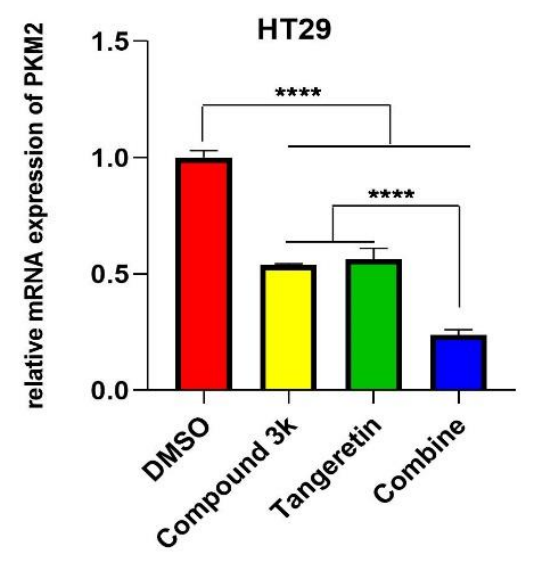

$\mathrm{E}$

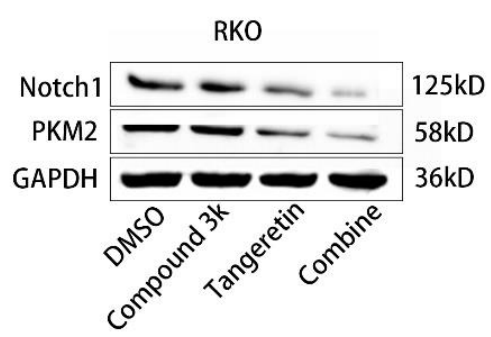

$\mathrm{H}$

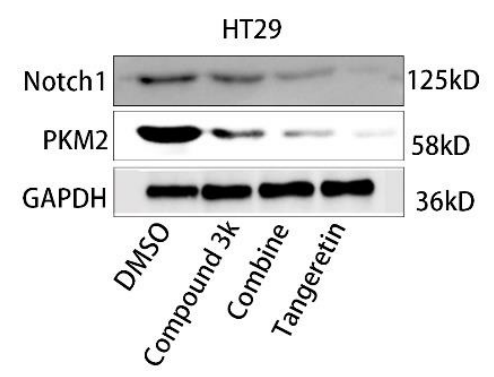

$\mathrm{D}$

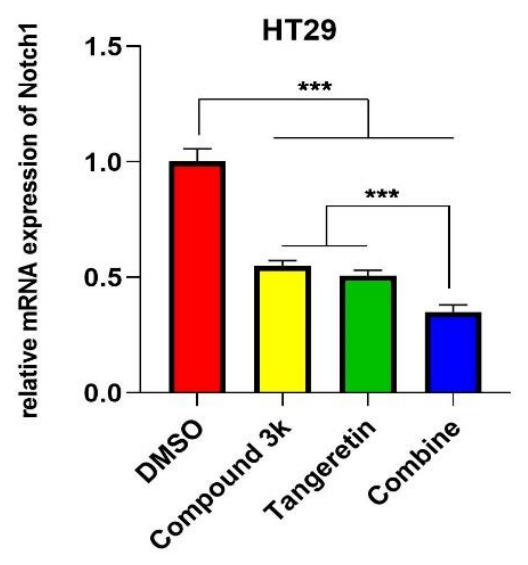

F

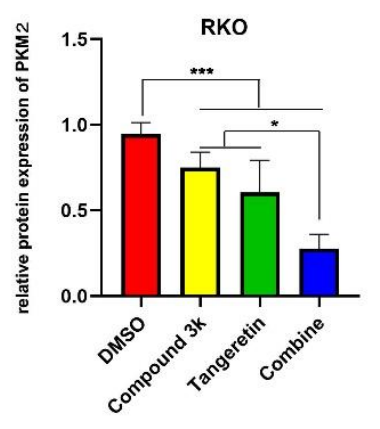

I

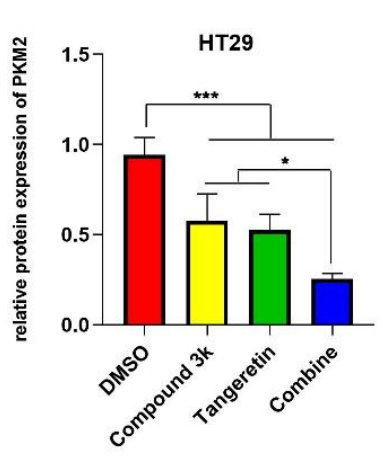

G

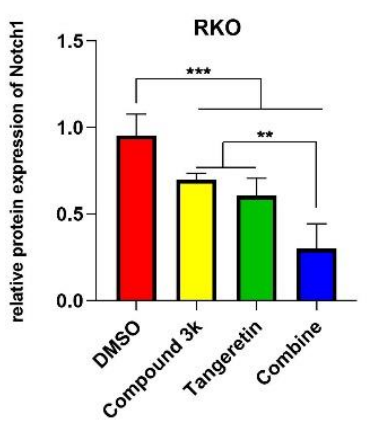

J

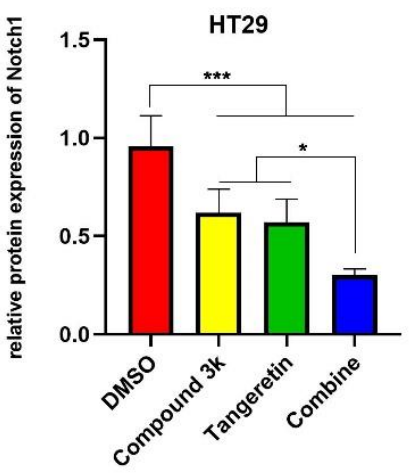

Figure 5. Expression of PKM2 and Notch1 in CRC cells treated with compound $3 \mathrm{~K}$ and TGN. (A-D) qRT-PCR was performed to detect the mRNA expression levels of PKM2 and Notch1 in the four groups of CRC cells after $24 \mathrm{~h}$ of treatment with DMSO (as a control), compound 3K, TGN, or the combination of compound 3K and TGN. (E-J) Western blot analysis was conducted to detect the expression of PKM2 and Notch1 in the four groups of CRC cells after 24 h of treatment. ${ }^{*} p<0.05 ; * * p<0.01 ; * * * p<0.001$. 


\section{Discussion}

$\mathrm{CRC}$ is one of the most common cancers and among the leading causes of cancerrelated mortality worldwide [8]. In addition, $\mathrm{CRC}$ is a highly proliferative and aggressive malignancy, resulting in poor patient prognosis. Excision and chemotherapy are the main treatment strategies for $\mathrm{CRC}$, although the benefits are limited due to side effects and recurrence [9]. Therefore, there is an urgent need to understand the mechanisms underlying the carcinogenesis of CRC and to develop new therapeutic strategies.

PKM2, which is upregulated in a various types of cancer, including CRC, catalyzes the final step of glycolysis and readjusts glycolytic flux to meet the specific metabolic needs of proliferating cells $[10,11]$. PKM2 promotes the mobility of gastric cancer cells via regulation of the PI3K/AKT pathway [12]. In prostate cancer, high expression of PKM2 during hypoxia leads to resistance to an mTOR inhibitor [13]. In CRC patients, high expression of PKM2 is predictive of a poor 5-year survival rate [5]. The Notch signaling pathway plays key roles in determining cell fate in multicellular organisms [14]. Abnormal expression of Notch1 has been attributed to the severity of CRC [15] and high Notch1 protein expression was correlated with a poor outcome in CRC [7]. Notch1 is also a prognostic biomarker of poorer survival of CRC patients [16]. Therefore, the aim of the present study was to determine the relationship between PKM2 and Notch1 in CRC.

Immunohistochemical analysis revealed high protein co-expression of PKM2 and Notch1 in CRC tissues. Moreover, as compared to NCM460 cells, the mRNA, and protein levels of PKM2 and Notch1 are highly expressed in RKO and HT29 cells. Targeting of PKM2 or Notch1 by gene silencing or pharmacological methods is an effective anti-cancer strategy. For example, the PKM2 inhibitor shikonin is especially effective against bladder cancer via the induction of necroptosis [17]. Another PKM2 inhibitor, compound $3 \mathrm{~K}$, has anti-proliferation effects in liver and colon cancers $[18,19]$. TGN, a Notch1-specific inhibitor, is effective against gastric cancer cells [20].

In the present study, the PKM2 inhibitor compound $3 \mathrm{~K}$ and the Notch1 inhibitor TGN were employed to determine the feasibility of simultaneous inhibition of PKM2 and Notch1 for treatment of CRC. The results showed that inhibition of PKM2 significantly decreased the expression of Notch1 in addition to the proliferation and mobility of CRC cells. Interestingly, inhibition of Notch1 had a notable effect on 
PKM2 expression and the proliferation and mobility of CRC cells. Therefore, the focus of this study was the relationship between PKM2 and Notch1. This study is the first to show that PKM2 and Notch1 form a positive feedback loop that promotes the development of CRC. In addition, PKM2 and Notch1 exerted synergistic inhibitory effects on the proliferation and mobility of CRC cells. Collectively, these results indicate that simultaneous inhibition of PKM2 and Notch1 presents a potentially effective strategy for treatment of CRC.

\section{Conclusion}

PKM2 and Notch1 form a positive feedback loop that promotes the development of CRC. The PKM2 inhibitor compound 3K and the Notch1 inhibitor TGN showed synergistic effects against the proliferation and migration of CRC cells. Therefore, simultaneous inhibition of PKM2 and Notch1 with compound $3 \mathrm{~K}$ and TGN, respectively, presents a novel and effective strategy for treatment of CRC.

\section{Availability of data and materials}

All data can be obtained from the manuscript or from request to the author.

\section{References}

[1] ARNOLD M, SIERRA M, LAVERSANNE M, et al. Global patterns and trends in colorectal cancer incidence and mortality [J]. Gut, 2017, 66(4): 683-91.

[2] ATTOUB S, ARAFAT K, KHALAF T, et al. Frondoside A Enhances the Anti-Cancer Effects of Oxaliplatin and 5-Fluorouracil on Colon Cancer Cells [J]. Nutrients, 2018, 10(5):

[3] CHEN J, XIE J, JIANG Z, et al. Shikonin and its analogs inhibit cancer cell glycolysis by targeting tumor pyruvate kinase-M2 [J]. Oncogene, 2011, 30(42): 4297-306.

[4] HACKER H, STEINBERG P, BANNASCH P. Pyruvate kinase isoenzyme shift from L-type to M2-type is a late event in hepatocarcinogenesis induced in rats by a choline-deficient/DL-ethioninesupplemented diet [J]. Carcinogenesis, 1998, 19(1): 99-107.

[5] CUI R, SHI X. Expression of pyruvate kinase M2 in human colorectal cancer and its prognostic value [J]. International journal of clinical and experimental pathology, 2015, 8(9): 11393-9.

[6] NING X, QI H, LI R, et al. Synthesis and antitumor activity of novel 2, 3-didithiocarbamate substituted naphthoquinones as inhibitors of pyruvate kinase M2 isoform [J]. Journal of enzyme inhibition and medicinal chemistry, 2018, 33(1): 126-9.

[7] CHU D, LI Y, WANG W, et al. High level of Notch1 protein is associated with poor overall survival in colorectal cancer [J]. Annals of surgical oncology, 2010, 17(5): 1337-42.

[8] FAVORITI P, CARBONE G, GRECO M, et al. Worldwide burden of colorectal cancer: a review [J]. Updates in surgery, 2016, 68(1): 7-11.

[9] SZARVAS T, CSIZMARIK A, NAGY N, et al. Molecular underpinnings of systemic treatment 
resistance in metastatic castration-resistant prostate cancer [J]. Orvosi hetilap, 2020, 161(20): 813-20.

[10] IQBAL M, GUPTA V, GOPINATH P, et al. Pyruvate kinase M2 and cancer: an updated assessment [J]. FEBS letters, 2014, 588(16): 2685-92.

[11] SHIROKI T, YOKOYAMA M, TANUMA N, et al. Enhanced expression of the M2 isoform of pyruvate kinase is involved in gastric cancer development by regulating cancer-specific metabolism [J]. Cancer science, 2017, 108(5): 931-40.

[12] WANG C, JIANG J, JI J, et al. PKM2 promotes cell migration and inhibits autophagy by mediating PI3K/AKT activation and contributes to the malignant development of gastric cancer [J]. Sci Rep, 2017, 7(1): 2886.

[13] YASUMIZU Y, HONGO H, KOSAKA T, et al. PKM2 under hypoxic environment causes resistance to mTOR inhibitor in human castration resistant prostate cancer [J]. Oncotarget, 2018, 9(45): 27698-707.

[14] ULVKLO C, MACDONALD R, BIVIK C, et al. Control of neuronal cell fate and number by integration of distinct daughter cell proliferation modes with temporal progression [J]. Development (Cambridge, England), 2012, 139(4): 678-89.

[15] TYAGi A, SHARMA A, DAMODARAN C. A Review on Notch Signaling and Colorectal Cancer [J]. Cells, 2020, 9(6).

[16] ARCAROLI J J, TAI W M, MCWILLIAMS R, et al. A NOTCH1 gene copy number gain is a prognostic indicator of worse survival and a predictive biomarker to a Notch1 targeting antibody in colorectal cancer [J]. Int J Cancer, 2016, 138(1): 195-205.

[17] WANG Y, HAO F, NAN Y, et al. PKM2 Inhibitor Shikonin Overcomes the Cisplatin Resistance in Bladder Cancer by Inducing Necroptosis [J]. International journal of biological sciences, 2018, 14(13): 1883-91.

[18] NING X, QI H, LI R, et al. Synthesis and antitumor activity of novel 2, 3-didithiocarbamate substituted naphthoquinones as inhibitors of pyruvate kinase M2 isoform [J]. Journal of enzyme inhibition and medicinal chemistry, 2018, 33(1): 126-9.

[19] ZENG Z, LAN J, LEI S, et al. Simultaneous Inhibition of Ornithine Decarboxylase 1 and Pyruvate Kinase M2 Exerts Synergistic Effects Against Hepatocellular Carcinoma Cells [J]. OncoTargets and therapy, 2020, 13(11697-709.

[20] ZHANG X, ZHENG L, SUN Y, et al. Tangeretin enhances radiosensitivity and inhibits the radiation-induced epithelial-mesenchymal transition of gastric cancer cells [J]. Oncology reports, 2015, 34(1): 302-10.

\section{Conflicts of Interest}

The authors have no conflicts of interest to declare.

\section{Ethical Statement}

The authors are accountable for all aspects of the work in ensuring that questions related to the accuracy or integrity of any part of the work are appropriately 
investigated and resolved. All procedures performed in studies involving human participants were in accordance with the ethical standards of the institutional and/or national research committee(s) and with the Helsinki Declaration (as revised in 2013). Written informed consent was obtained from the patient.

\section{Funding}

This study was supported by Natural Science Foundation of Gansu Province( No.1606RJZA198) and Science and Technology Development Program Project of Xigu.

\section{Consent for publication}

Not applicable.

\section{Authors' contributions}

JW, PB and WD wrote the original manuscript and conducted the experiments; MS and YG design the study and reviewed the draft; JP, BK and YJ collected the data and conducted analysis; BL and LY collected data and reviewed manuscript. All authors read and approved the final manuscript.

\section{Acknowledgements}

Not applicable. 